International Mathematical Forum, 1, 2006, no. 40, 1975-1982

\title{
Generalized Quasilinearization Method for the Forced Düffing Equation with Mixed B.C.
}

\author{
Ramzi S. N. Alsaedi \\ Department of Mathematics \\ King Abdul Aziz University \\ Jeddah P.O.Box 80203, Saudia Arabia \\ ramzialsaedi@yahoo.co.uk
}

\begin{abstract}
A generalized quasilinearization method for the nonlinear forced Düffing equation with mixed boundary conditions is developed and a sequence of approximate solutions converging monotonically and quadratically to the solution of the given mixed boundary value problem is presented.
\end{abstract}

Mathematics Subject Classification: 34A45, 34B15

Keywords: Quasilinearization; Forced Düffing equation; Mixed B.C.; Quadratic convergence

\section{Introduction}

The method of quasilinearization [2] provides an excellent approach for obtaining approximate solutions of nonlinear differential equations. This technique works fruitfully only for the problems involving convex/concave functions and gives the sequence of approximate solutions converging monotonically and quadratically to the solution. Later after that the convexity assumption was relaxed and the method was generalized and extended in various directions to make it applicable to a large class of problems [5-9]. The generalized quasilinearization method was discussed for second order boundary value problems [11-14]. A generalized quasilinearization method was developed for a second order ordinary nonlinear differential equation with mixed boundary conditions $[1]$.

The Düffing equation is a well known nonlinear equation of applied science which is used as a powerful tool to discuss some important practical 
phenomena. In this paper the aim is to consider and study a second order ordinary nonlinear differential equation, namely, the forced Düffing equation with mixed boundary value conditions without requiring the nonlinear force function involved to be convex/concave, and obtain a sequence of approximate solutions converging quadratically to a solution of the problem. Moreover, this paper shows that, if the damping part in the forced Düffing equation vanishes, then this will give [1] as a special case.

\section{Preliminaries}

The Düffing equation with mixed B.C.

$$
\begin{aligned}
-\psi^{\prime \prime}(t)-k \psi^{\prime}(t) & =\lambda \psi(t), \quad t \in J=[0, \pi] \\
\psi(0) & =\psi^{\prime}(\pi)=0,
\end{aligned}
$$

has a nontrivial solution if and only if $\gamma=\frac{1}{\pi} \cot ^{-1}\left(\frac{-\delta}{\gamma}\right)$, where $\gamma=\frac{\sqrt{4 \lambda-k^{2}}}{2}$ and $\delta=\frac{-k}{2}$, where $k \in R$. If $k=0$, then the above mixed boundary value problem has a nontrivial solution if and only if $\lambda=[(2 m-1) / 2]^{2}(m=1,2,3 \cdots)$ as shown in [1]. For $\gamma \neq \frac{1}{\pi} \cot ^{-1}\left(\frac{-\delta}{\gamma}\right)$ and $\omega(t) \in C[0, \pi]$, the unique solution of the mixed boundary value problem

$$
\begin{aligned}
-\psi^{\prime \prime}(t)-k \psi^{\prime}(t)-\lambda \psi & =\omega(t), \quad t \in J=[0, \pi] \\
\psi(0) & =\psi^{\prime}(\pi)=0
\end{aligned}
$$

is given by

$$
\psi(t)=\int_{0}^{\pi} G_{\lambda}(t, v) \omega(v) d v .
$$

Here, $G_{\lambda}(t, v)$ is the Green's function, where $G_{\lambda}(t, v)$ for $\lambda>\frac{k^{2}}{4}$, is given by

$$
\begin{aligned}
& {\left[e^{\delta \pi}(\delta \sin \gamma \pi+\gamma \cos \gamma \pi)\right]^{-1} e^{\delta(v+\pi-t)} \sin \gamma v } \\
& \times\left[\cos \gamma(\pi-t)-\frac{\delta}{\gamma} \sin \gamma(\pi-t)\right], \\
0 \leq & v \leq t \leq \pi \\
& {\left[e^{\delta \pi}(\delta \sin \gamma \pi+\gamma \cos \gamma \pi)\right]^{-1} e^{\delta(t+\pi-v)} \sin \gamma t } \\
& \times\left[\cos \gamma(\pi-v)-\frac{\delta}{\gamma} \sin \gamma(\pi-v)\right], \\
0 \leq & t \leq v \leq \pi
\end{aligned}
$$


And $G_{\lambda}(t, v)$ for $\lambda<\frac{k^{2}}{4}$, is given by

$$
\begin{aligned}
& {\left[e^{\delta \pi}(\delta \sinh \gamma \pi+\gamma \cosh \gamma \pi)\right]^{-1} e^{\delta(v+\pi-t)} \sinh \gamma v } \\
& \times\left[\cosh \gamma(\pi-t)-\frac{\delta}{\gamma} \sinh \gamma(\pi-t)\right], \\
0 \leq & v \leq t \leq \pi, \\
& {\left[e^{\delta \pi}(\delta \sinh \gamma \pi+\gamma \cosh \gamma \pi)\right]^{-1} e^{\delta(t+\pi-v)} \sinh \gamma t } \\
& \times\left[\cosh \gamma(\pi-v)-\frac{\delta}{\gamma} \sinh \gamma(\pi-v)\right], \\
0 \leq & t \leq v \leq \pi .
\end{aligned}
$$

When $\lambda=\frac{k^{2}}{4}$, then $G_{\frac{k^{2}}{4}}(t, v)$ is given by

$$
\begin{aligned}
& {[(1+\delta \pi)(\cosh \delta \pi+\sinh \delta \pi)]^{-1}[v(\cosh \delta v+\sinh \delta v) \cosh \delta(\pi-t)] } \\
0 \leq & v \leq t \leq \pi \\
& {[(1+\delta \pi)(\cosh \delta \pi+\sinh \delta \pi)]^{-1}[t(\cosh \delta t+\sinh \delta t) \cosh \delta(\pi-v)] } \\
0 \leq & t \leq v \leq \pi
\end{aligned}
$$

Note that, putting $k=0$ in the given mixed BVP, then this will give [1] as a special case.

Now, consider the following nonliner mixed BVP

$$
\begin{gathered}
-u^{\prime \prime}(t)-k u^{\prime}(t)=f(t, u(t)), \quad t \in J=[0, \pi], \\
u(0)=u^{\prime}(\pi)=0,
\end{gathered}
$$

where $f: J \times R \rightarrow R$ is a continuouse real valued function. A function $\alpha \in C^{2}[J, R]$ is a lower solution of (2.1) if

$$
\begin{aligned}
-\alpha^{\prime \prime}(t)-k \alpha^{\prime}(t) & \leqslant f(t, \alpha(t)), \quad t \in J \\
\alpha(0) & \leqslant 0, \alpha^{\prime}(\pi) \leqslant 0 .
\end{aligned}
$$

Analogously, $\beta \in C^{2}[J, R]$ is an upper solution of (2.1) if

$$
\begin{aligned}
-\beta^{\prime \prime}(t)-k \beta^{\prime}(t) & \geqslant f(t, \beta(t)), \quad t \in J, \\
\beta(0) & \geqslant 0, \beta^{\prime}(\pi) \geqslant 0 .
\end{aligned}
$$

The following lemma plays a crucial role in the sequel and we sketch its proof for the sake of completeness.

Lemma 2.1. Assume that $\alpha, \beta \in C^{2}[J, R]$ are lower and upper solutions of (2.1), respectively, such that $\alpha(t) \leqslant \beta(t)$ for every $t \in J$. Then there exists a solution $u(t)$ of $(2.1)$ such that $\alpha(t) \leqslant u(t) \leqslant \beta(t)$ for $t \in J$. 
Proof. Let $p: J \times R \rightarrow R$ be a mapping defined by

$$
p(t, u)=\max \{\alpha(t), \min \{u(t), \beta(t)\}\} .
$$

Then, extend $f(t, u(t))$ to $J \times R$ by setting $F(t, u(t))=f(t, p(t, u(t)))$.

Observe that $F(t, u(t))$ is bounded. Let us consider the modified (2.1) BVP

$$
\begin{gathered}
-u^{\prime \prime}(t)-k u^{\prime}(t)=F(t, u(t)), \quad t \in J, \\
u(0)=u^{\prime}(\pi)=0,
\end{gathered}
$$

which is solvable, that is $(2.2)$ has a solution of $u(t)$ on $J$. Using the arguments similar to those of $[4,11]$, it can be shown that

$\alpha(t) \leqslant u(t) \leqslant \beta(t)$ on $J$.

Finally, since any solution of (2.2) is also a solution of (2.1), it follows that $u(t)$ is a solution of $(2.1)$.

\section{Main result}

Theorem 3.1. Assume that

$\left(\mathrm{A}_{1}\right) \alpha_{0}, \beta_{0} \in C^{2}[J, R]$ are lower and upper solutions of (2.1), respectively, such that $\alpha_{0}(t) \leqslant \beta_{0}(t)$ on $J$,

$\left(\mathrm{A}_{2}\right) f \in C[\Omega, R]$ is such that $f_{u}(t, u), f_{u u}(t, u)$ exist and are continuous for every $(t, u) \in \Omega$, where

$$
\Omega=\left\{(t, u) \in J \times R: \alpha_{0}(t) \leqslant u(t) \leqslant \beta_{0}(t)\right\},
$$

$\left(\mathrm{A}_{3}\right) \quad f_{u}(t, u)<0$ for every $(t, u) \in \Omega$.

Then there exists monotone nondecreasing sequence $\left\{\alpha_{n}\right\}$ which converges uniformly to a solution of (2.1) and the convergence is quadratic.

Proof. Let $F: J \times R \rightarrow R$ is such that $F(t, u), F_{u}(t, u)$ and $F_{u u}(t, u)$ are continuous on $J \times R$ and

$$
F_{u u}(t, u) \geqslant 0, \quad(t, u) \in J \times R .
$$

Motivated by Eloe and Zhang [3], take $\Phi(t, u)=F(t, u)-f(t, u)$ on $J \times R$. In view of (3.1), we see that

$$
F(t, u) \geqslant F(t, v)+F_{u}(t, v)(u-v)
$$

for $u \geqslant v$ and therefore

$$
f(t, u) \geqslant f(t, v)+F_{u}(t, v)(u-v)-[\Phi(t, u)-\Phi(t, v)] .
$$

Now, consider the mixed BVP

$$
\begin{gathered}
-u^{\prime \prime}(t)-k u^{\prime}(t)=g\left(t, u ; \alpha_{0}\right)=f\left(t, \alpha_{0}\right)+F_{u}\left(t, \alpha_{0}\right)\left(u-\alpha_{0}\right) \\
-\left[\Phi(t, u)-\Phi\left(t, \alpha_{0}\right)\right]
\end{gathered}
$$




$$
u(0)=u^{\prime}(\pi)=0 .
$$

The inequality (3.2) and $\left(\mathrm{A}_{1}\right)$ imply

$$
\begin{aligned}
-\alpha_{0}^{\prime \prime}(t)-k \alpha_{0}^{\prime}(t) \leqslant & f\left(t, \alpha_{0}(t)\right)=g\left(t, \alpha_{0} ; \alpha_{0}\right) \\
-\beta_{0}^{\prime \prime}(t)-k \beta_{0}^{\prime}(t) \geqslant & f\left(t, \beta_{0}(t)\right) \geqslant f\left(t, \alpha_{0}\right)+F_{u}\left(t, \alpha_{0}\right)\left(\beta_{0}-\alpha_{0}\right) \\
& -\left[\Phi\left(t, \beta_{0}\right)-\Phi\left(t, \alpha_{0}\right)\right] \\
= & g\left(t, \beta_{0} ; \alpha_{0}\right) .
\end{aligned}
$$

By lemma 2.1, there exists a solution $\alpha_{1}$ of (3.3) such that $\alpha_{0}(t) \leqslant \alpha_{1}(t) \leqslant \beta_{0}(t)$ on $J$. Next, consider the mixed BVP

$$
\begin{gathered}
-u^{\prime \prime}(t)-k u^{\prime}(t)=g\left(t, u ; \alpha_{1}\right), \\
u(0)=u^{\prime}(\pi)=0 .
\end{gathered}
$$

Observe that

$$
\begin{aligned}
-\alpha_{1}^{\prime \prime}(t)-k \alpha_{1}^{\prime}(t)= & g\left(t, \alpha_{1} ; \alpha_{0}\right) \\
= & f\left(t, \alpha_{0}\right)+F_{u}\left(t, \alpha_{0}\right)\left(\alpha_{1}-\alpha_{0}\right)-\left[\Phi\left(t, \alpha_{1}\right)-\Phi\left(t, \alpha_{0}\right)\right] \\
\leqslant & f\left(t, \alpha_{1}\right)=g\left(t, \alpha_{1} ; \alpha_{1}\right), \\
-\beta_{0}^{\prime \prime}(t)-k \beta_{0}^{\prime}(t) \geqslant & f\left(t, \beta_{0}(t)\right) \geqslant f\left(t, \alpha_{1}\right)+F_{u}\left(t, \alpha_{1}\right)\left(\beta_{0}-\alpha_{1}\right) \\
& -\left[\Phi\left(t, \beta_{0}\right)-\Phi\left(t, \alpha_{1}\right)\right] \\
= & g\left(t, \beta_{0} ; \alpha_{1}\right),
\end{aligned}
$$

in view of (3.2). It follows from lemma 2.1 that there exists a solution $\alpha_{2}$ such that $\alpha_{1}(t) \leqslant \alpha_{2}(t) \leqslant \beta_{0}(t)$ on $J$. Thus, $\alpha_{0}(t) \leqslant \alpha_{1}(t) \leqslant \alpha_{2}(t) \leqslant \beta_{0}(t)$ on $J$. Employing the same arguments successively, we conclude

$$
\alpha_{0}(t) \leqslant \alpha_{1}(t) \leqslant \alpha_{2}(t) \cdots \leqslant \alpha_{n}(t) \leqslant \beta_{0}(t) \quad \text { on } J
$$

where the elements of the monotone sequence $\left\{\alpha_{n}(t)\right\}$ are the solutions of the mixed BVP

$$
\begin{aligned}
-u^{\prime \prime}-k u^{\prime}= & g\left(t, u ; \alpha_{n-1}\right)=f\left(t, \alpha_{n-1}\right)+F_{u}\left(t, \alpha_{n-1}\right)\left(u-\alpha_{n-1}\right) \\
& -\left[\Phi(t, u)-\Phi\left(t, \alpha_{n-1}\right)\right] \\
u(0)= & u^{\prime}(\pi)=0 .
\end{aligned}
$$

The monotonicity of the sequence $\left\{\alpha_{n}(t)\right\}$ ensures the existence of its (pointwise) limit $u$. 
Let us consider the linear mixed BVP

$$
\begin{gathered}
-u^{\prime \prime}(t)-k u^{\prime}(t)-\frac{k^{2}}{4} u(t)=f_{n}(t), \\
u(0)=u^{\prime}(\pi)=0,
\end{gathered}
$$

where

$$
f_{n}(t)=g\left(t, \alpha_{n}(t) ; \alpha_{n-1}(t)\right), \quad \text { on } t \in J .
$$

The continuity of $g$ on $\Omega$ implies that the sequence $\left\{f_{n}\right\}$ is bounded in $C[J, R]$ and so

$$
\lim _{n \rightarrow \infty} f_{n}(t)=f(t, u(t)), \quad t \in J
$$

Here

$$
\alpha_{n}(t)=\int_{0}^{\pi} G_{\frac{k^{2}}{4}}(t, s) f_{n}(s) d s,
$$

where $\alpha_{n}(t)$ is a solution of (3.5). Thus $\left\{\alpha_{n}(t)\right\}$ is bounded in $C^{2}[J, R]$ and $\left\{\alpha_{n}(t)\right\} \uparrow u$ uniformly on $J$. Consequently,

$$
u(t)=\int_{0}^{\pi} G_{\frac{k^{2}}{4}}(t, s) f(s, u(s)) d s, \quad t \in J .
$$

Hence $u$ is a solution of (2.1).

For quadratic convergence, we set the error as $p_{n}(t)=u(t)-\alpha_{n}(t)$. Using the mean value theorem repeatedly, we obtain

$$
\begin{aligned}
-p_{n}^{\prime \prime}(t)-k p_{n}^{\prime}(t)= & f(t, u(t))-g\left(t, \alpha_{n}(t) ; \alpha_{n-1}(t)\right) \\
= & f(t, u(t))-f\left(t, \alpha_{n-1}(t)\right)-F_{u}\left(t, \alpha_{n-1}(t)\right)\left[\alpha_{n}(t)-\alpha_{n-1}(t)\right] \\
& +\left[\Phi\left(t, \alpha_{n}(t)\right)-\Phi\left(t, \alpha_{n-1}(t)\right)\right] \\
= & F(t, u(t))-F\left(t, \alpha_{n-1}(t)\right)-F_{u}\left(t, \alpha_{n-1}(t)\right)\left[\alpha_{n}(t)-\alpha_{n-1}(t)\right] \\
& +\left[\Phi\left(t, \alpha_{n}(t)\right)-\Phi(t, u(t))\right] \\
= & F_{u}(t, \xi)\left[u(t)-\alpha_{n-1}(t)\right]-F_{u}\left(t, \alpha_{n-1}(t)\right)\left[\alpha_{n}(t)-\alpha_{n-1}(t)\right] \\
& +\left[\Phi\left(t, \alpha_{n}(t)-\Phi(t, u(t))\right]\right. \\
= & {\left[F_{u}(t, \xi)-F_{u}\left(t, \alpha_{n-1}(t)\right)\right]\left[u(t)-\alpha_{n-1}(t)\right]+F_{u}\left(t, \alpha_{n-1}(t)\right) } \\
& \times\left[u(t)-\alpha_{n}(t)\right]+\left[\Phi\left(t, \alpha_{n}(t)\right)-\Phi(t, u(t))\right] \\
= & F_{u u}(t, \sigma)\left[\xi-\alpha_{n-1}(t)\right]\left[u(t)-\alpha_{n-1}(t)\right]+F_{u}\left(t, \alpha_{n-1}(t)\right) \\
& \times\left[u(t)-\alpha_{n}(t)\right]-\Phi_{u}(t, \eta)\left[u(t)-\alpha_{n}(t)\right]
\end{aligned}
$$

where

$$
\alpha_{n-1}(t) \leqslant \xi \leqslant \sigma \gtrless u(t) \text { and } \alpha_{n}(t) \leqslant \eta \gtrless u(t)
$$


Set

$$
h_{n}(t)=F_{u}\left(t, \alpha_{n-1}(t)\right)-\Phi_{u}(t, \eta),
$$

and

$$
l_{n}(t)=F_{u u}(t, \sigma)\left[\xi-\alpha_{n-1}(t)\right]\left[u(t)-\alpha_{n-1}(t)\right]-M p_{n-1}^{2}(t),
$$

where $0 \leqslant F_{u u}(t, v) \leqslant M,(t, v) \in \Omega$. Clearly $l_{n}(t) \leqslant 0$. Since $F_{u}$ is nondecreasing and $\alpha_{n-1}(t) \leqslant \eta$, it follows by $\left(\mathrm{A}_{3}\right)$ that there exists $\lambda<\frac{k^{2}}{4}$ and an integer $N$ such that $h_{n}(t) \leqslant \lambda, t \in J$ for $n \geqslant N$. thus the error $p_{n}$ satisfies the mixed BVP

$$
\begin{aligned}
-p_{n}^{\prime \prime}(t)-k p_{n}^{\prime}(t)-\lambda p_{n}(t) & =\left[h_{n}(t)-\lambda\right] p_{n}(t)+M p_{n-1}^{2}(t)+l_{n}(t) \\
p_{n}(0) & =p_{n}^{\prime}(\pi)=0 .
\end{aligned}
$$

This implies that

$$
p_{n}(t)=\int_{0}^{\pi} G_{\lambda}(t, s)\left(\left[h_{n}(s)-\lambda\right] p_{n}(s)+M p_{n-1}^{2}(s)+l_{n}(s)\right) d s,
$$

which gives

$$
p_{n}(t) \leqslant M \int_{0}^{\pi} G_{\lambda}(t, s) p_{n-1}^{2}(s) d s, \quad n \geqslant N .
$$

Hence, there exists a constant $\delta>0$ such that

$$
\left\|p_{n}\right\| \leqslant \delta\left\|p_{n-1}\right\|^{2}, \quad n \geqslant N,
$$

where $\|u\|=\max \{|x(t)|: t \in J\}$ is the usual uniform norm on $C[J, R]$.

Acknowledgement. This research is completed while the author was awarded the Kuwait Junior Research Fellowship at the Department of Pure Mathematics and Mathematical Statistics, at the University of Cambridge, by the Kuwait Foundation for the Advancement of Sciences, for the six months period during February, 2006 to August, 2006, so the author would like to express his sincere thanks and gratitude to the Kuwait Foundation for the Advancement of Sciences and to the Department of Pure Mathematics and Mathematical Statistics, at the University of Cambridge.

\section{References}

[1] B. Ahmad, J.J. Nieto, N. Shahzad, Generalized quasilinearization for mixed boundary value problems, Appl. Math. Comp., 133 (2002), 423429. 
[2] R. Bellman, R. Kalaba, Quasilinearization and nonlinear boundary value problem, Elsevier, New York, 1965.

[3] P.W. Eloe, Y. Zhang, A quadratic monotone iteration scheme for twopoint boundary value problem for ordinary differential equations, Nonlinear Anal., 33 (1988), 443-453.

[4] G.S. Ladde, V. Lakshmikantham, A.S. Vatsala, Monotone iteration techniques for nonlinear differential equations, Pitman, Boston, MA, 1985.

[5] V. Lakshmikantham, An extension of the method of quasilinearization, J. Optim. Theory Appl., 82 (1994), 315-321.

[6] V. Lakshmikantham, Further improvement of generalized quasilinearization, Nonlinear Anal., 27 (1996), 223-227.

[7] V. Lakshmikantham, S. Leela, F.A. McRae, Improved generalized quasilinearization method, Nonlinear Anal., 24 (1995), 1627-1637.

[8] V. Lakshmikantham, N. Shahzad, Further generalization of generalized quasilinearization method, J. Appl. Math. Stochastics Anal., 7 (1994), 545-552.

[9] V. Lakshmikantham, N. Shahzad, J.J. Nieto, Method of generalized quasilinearization for periodic boundary value problems, Nonlinear Anal., 27 (1996), 143-151.

[10] V. Lakshmikantham, A.S. Vatsala, Generalized quasilinearization for nonlinear problems, Kluwer Academic Publishers, Boston., (1998).

[11] J.J. Nieto, Generalized quasilinearization method for a second order ordinary differential equation with Dirichlet boundary coditions, Proc. Amer. Math. Soc., 125 (1997), 2599-2604.

[12] N. Shahzad, S. Sivasundram, Extended quasilinearization method for boundary value problems, Nonlinear World., 2 (1995), 311-319.

[13] N. Shahzad, A.S. Vatsala, Extension of the method of generalized quasilinearization for second order boundary value problems, Appl. Anal., 58 (1995), 77-83.

[14] N. Shahzad, A.S. Vatsala, Improved generalized quasilinearization method for second order boundary value problems, Dyn. Syst. Appl., 4 (1995), 79-85.

\section{Received: April 26, 2006}

